\title{
A survey of visual impairment in children attending the Royal Blind School, Edinburgh using the WHO childhood visual impairment database
}

\begin{abstract}
Purpose To assess the aetiology and changing patterns of childhood blindness in one school for the blind in the UK and to assess the use of the World Health Organisation Prevention of Blindness (WHO/PBL) methodology and reporting form in a developed country.

Methods One hundred and seven children in one school for the blind and visually impaired in Edinburgh were examined using the WHO/PBL childhood blindness assessment form.

Results Of the 107 children examined, 87 $(81 \%)$ were blind or severely visually impaired (corrected visual acuity of $<6 / 60$ $(20 / 200)$ in the better eye). Perinatal related blindness $(40 \%)$, hereditary disease $(26 \%)$ and developmental factors $(26 \%)$ formed the three largest aetiological categories. Conclusion The pattern of childhood blindness seen in this study was similar to reports from other developed countries. The WHO/PBL reporting form allows detailed comparisons between countries and over time. Additional fields for more detailed reporting of cerebral visual impairment and associated handicaps would increase the usefulness of the WHO/PBL form for population-based studies and for use in developed countries.

Eye (2002) 16, 557-561. doi:10.1038/

sj.eye. 6700149
\end{abstract}

Keywords: childhood blindness; congenital cataract; hereditary blindness; microphthalmos; retinal dystrophies; retinopathy of prematurity

J Alagaratnam, TK Sharma, CS Lim and

BW Fleck

Introduction

The prevalence and major causes of childhood blindness vary between countries and over time. The WHO Prevention of Blindness Program with the International Centre for Eye Health has developed a standard methodology and reporting form to record the causes of visual impairment in children. ${ }^{1}$ The methodology has been used in developing and middle income countries. ${ }^{1-3}$

This study was undertaken in order to assess this method in one school for blind children in the UK. There have been two previous surveys of visual impairment in The Royal Blind School, Edinburgh. ${ }^{4,5}$ These studies and studies from other developed countries ${ }^{6}$ were presented in a nonstandardised way, which makes it difficult to monitor changing patterns of childhood blindness over time.

The aims of this study were to apply the WHO/PBL reporting form in a UK school for the blind and to compare the findings with data from schools in developing countries.

Patients and methods

The Royal Blind School, Edinburgh serves Scotland and the North East of England. The school caters for both residential and day students. Many blind children are now educated in their local school with additional educational support and the role of special schools for blind children has changed in the UK. Since 1990, the Royal Blind School Edinburgh has been divided into two sites with nursery, primary and secondary education at the main school site and a
Department of Ophthalmology Royal Infirmary Edinburgh, UK

Place of Study: Royal Blind Schoo Craigmillar Park Edinburgh, UK

Correspondence: BW Fleck

Department of Ophthalmology Princess Alexandra Eye Pavilion Chalmers Street Edinburgh EH3 9HA, UK Tel: 01315363905 Fax: 01315363735 E-mail: Brian.Fleck@ luht.scot.nhs.uk

Financial or proprietary interest: none 
residential, multiple handicap unit (MHU) at a second site.

During the academic year 1999-2000, all 107 children at the school were examined by one consultant ophthalmologist (BWF). Information was gathered using interviews with pupils, parents and school staff and by consulting hospital and community health records for each child.

Binocular visual acuity, and when possible, uniocular visual acuity were measured using a range of techniques. These included Keeler grating cards, Cardiff cards, Kay pictures, Sheridan-Gardinar test cards, and Snellen charts. Visual fields were assessed by confrontation, and by the history given by carers. Refraction with cycloplegia was carried out. Anterior segment examination was performed using a slit-lamp or flashlight. Posterior segment examination was performed by indirect ophthalmoscopy after mydriasis.

All data were recorded on the WHO/PBL eye examination record for children with blindness and low vision. The form is accompanied by instructions for use, definitions and methods of classification. For each child, the cause of visual loss was recorded using the anatomical and aetiological classification used in the form.

\section{Results}

One hundred and seven children were seen. Sixty-six were attending the main school campus and 41 the MHU. There were 54 males and 53 females. Two children were of Middle Eastern origin, one was Chinese, one was from Pakistan and all the remaining children were Caucasian. Seventy-five $(70 \%)$ of the children were aged 11 years or over, 31 (29\%) were aged 5-10 years, and one (1\%) was less than 5 years. The distribution of visual acuities is given in Table 1 . Within the group of 42 blind children with visual acuity $<3 / 60$ but with at least perception of light vision, 22 had detectable residual visual behaviour and

Table 1 WHO categories of visual impairment. Distribution of binocular visual acuity

\begin{tabular}{llcc}
\hline WHO category & Visual acuity & $\begin{array}{c}\text { Total } \\
\text { number }\end{array}$ & $\begin{array}{c}\text { Total } \\
\text { percentage }\end{array}$ \\
\hline Blind & NPL & 30 & $28 \%$ \\
Blind & $<3 / 60-\mathrm{PL}$ & 42 & $39 \%$ \\
Severe visual & $<6 / 60$ to $3 / 60$ & 15 & $14 \%$ \\
impairment & & & \\
Visual impairment & $<6 / 18$ to $6 / 60$ & 18 & $17 \%$ \\
No impairment & $6 / 18$ or better & 2 & $2 \%$ \\
Total & & 107 & $100 \%$ \\
\hline
\end{tabular}

NPL $=$ No perception of light. PL $=$ Perception of light.
20 had no functional vision. This latter group showed response to light or even movements of large objects during testing but showed no evidence of visual behaviour during daily activities. A total of 57 children therefore had residual vision and the remaining 50 had no functionally useful vision. Two children had visual acuity of $6 / 18$ or better. These children had profound perceptual visual dysfunction.

\section{Anatomical cause of visual loss}

The anatomical sites of abnormality leading to visual impairment or blindness are given in Table 2. Disease or malformation of the central nervous system or optic nerve was the commonest cause of visual impairment and was present in $53(50 \%)$ children. Combined optic atrophy and cerebral visual impairment were seen in 28 children. Seven children had cerebral visual impairment without optic atrophy. Optic atrophy was responsible for visual impairment in nine children and bilateral optic nerve hypoplasia in nine children.

Retinal disease was the second most common cause of visual impairment, present in 39 (36\%) children. Nineteen children had bilateral retinal detachment due to retinopathy of prematurity. Retinal dystrophies were present in 13 children, of whom seven were diagnosed as Leber's amaurosis. Two children had been treated for bilateral retinoblastoma.

Congenital anomalies of the whole globe, such as anophthalmos and microphthalmos, were present in 12 (11\%) children. Corneal opacity was relatively uncommon. One child had sclerocornea. There were two children with visual loss due to congenital glaucoma. There were no cases of visual impairment due to congenital cataract.

\section{Aetiological category of visual loss}

The causes of visual impairment are classified according to the time of known insult in Table 3. Ninety-one $(85 \%)$ had visual impairment by the age of

Table 2 Anatomical classification of the causes of visual impairment

\begin{tabular}{lcc}
\hline Anatomical site & $n$ & $\%$ \\
\hline Optic nerve/CNS & 53 & $50 \%$ \\
Retina & 39 & $36 \%$ \\
Whole globe & 12 & $11 \%$ \\
Glaucoma & 2 & $2 \%$ \\
Cornea & 1 & $1 \%$ \\
Lens & - & - \\
Uvea & - & - \\
Total & 107 & $100 \%$ \\
\hline
\end{tabular}


Table 3 Causes of visual impairment by timing of aetiological insult

\begin{tabular}{lrr}
\hline Timing of aetiological insult & $n$ & $\%$ \\
\hline Perinatal & 43 & $40 \%$ \\
Hereditary & 28 & $26 \%$ \\
Intrauterine & 3 & $3 \%$ \\
Childhood & 5 & $5 \%$ \\
Others ('Developmental') & 28 & $26 \%$ \\
Total & 107 & $100 \%$ \\
\hline
\end{tabular}

1 year and, of these, at least 48 (45\%) had visual impairment at birth.

Perinatal factors were identified in 43 (40\%) children, with prematurity the largest single group. Visual impairment was attributed to genetic diseases in 28 (26\%) children, most commonly optic atrophy or retinal dystrophy. Parental consanguinity was present in only one family. Intrauterine factors such as congenitally acquired rubella and congenital toxoplasmosis was present in only three (3\%) children. In the childhood onset group trauma or tumour had occurred in five (5\%) children. Developmental factors contributed to visual impairment in $28(26 \%)$ children. In many of these genetic abnormalities undetectable by current techniques may have been present.

\section{Additional impairment}

Table 4 summarises impairments present in addition to visual impairment. Only 25 (23\%) children had no additional impairment. Mental retardation was the most common defined additional impairment, present in $29(27 \%)$ children. Cerebral visual impairment was the most common cause of visual impairment in the school. Many of the children had multiple impairments due to brain injury, with cerebral palsy, mental retardation and epilepsy. Eight $(7.5 \%)$ children had hearing impairment.

\section{Discussion}

Childhood blindness has been identified as a priority in the World Health Organisation's global initiative for

Table 4 Additional disability associated with visual impairment

\begin{tabular}{lcc}
\hline Additional disability & $n$ & $\%$ \\
\hline None & 25 & $23 \%$ \\
Mental retardation & 29 & $27 \%$ \\
Epilepsy & 24 & $22 \%$ \\
Physical handicap & 21 & $19.65 \%$ \\
Hearing loss & 8 & $7.5 \%$ \\
\hline
\end{tabular}

the elimination of avoidable blindness by year $2020 .^{7}$ It has been estimated that there are 1.5 million severely visually impaired and blind children worldwide. The recorded prevalence of SVI/BL in European countries varies from 0.1 to 0.41 per 1000 children. ${ }^{6,8}$ There are a number of blind school studies from South Asia, ${ }^{2,3}$ South America ${ }^{9}$ and Africa. ${ }^{10,11}$ The causes of blindness vary from country to country and over time. There are few data available concerning the causes of childhood blindness in Scotland and a variety of definitions and classifications have been used in previous studies. ${ }^{4,5}$ This blind school study using the WHO/PBL eye examination form is the first reported in the United Kingdom.

This is a small study in a selected population and the results should be interpreted with caution. Integrated education of visually impaired children is now preferred when possible. A range of uncontrolled factors such as an increased prevalence of multiple handicaps and increased severity of visual impairment may have led to population bias in this study. The study results are therefore likely to differ from whole population studies.

The commonest anatomical site of disease was the brain and optic nerve. While it can be difficult to differentiate brain and optic nerve disease, some attempt to do this is helpful. The combining of optic nerve disease and Central Nervous System disease in the anatomical site category of the WHO/PBL data form should perhaps be reviewed. In this study 28 children had combined cerebral blindness and optic atrophy, seven had cerebral blindness alone, nine had optic atrophy, and nine had optic nerve hypoplasia.

Eighty-two children had a significant associated additional disability. The WHO/PBL data forms have a limited number of disability categories available. Mental retardation, epilepsy and physical handicap were present in 29, 24 and 21 children respectively. There was much overlap of these groups and the finding of co-existence of cerebral visual impairment and cerebral palsy was frequent. Distinct categories of cerebral visual impairment are now recognised. ${ }^{12,13}$ While a simple breakdown of associated disability may be appropriate for use in developing countries, a rather more sophisticated approach to cerebral visual impairment and associated impairments would be useful in developed countries. Studies in blind schools in developing countries have had a selection bias towards ocular disease. ${ }^{14,15}$ While the presence of a multiple handicap unit within the Royal Blind School, Edinburgh reduced this bias, it is likely that some bias towards ocular disease remained.

The relative frequency of genetic and developmental disorders and the rarity of infectious and nutritional 
conditions was similar to reports from other developed countries. ${ }^{16,17}$ Overall, $26 \%$ of children had visual impairment attributable to genetic disease. The mode of inheritance in the majority of these children was probably autosomal recessive. Retinal dystrophies (Leber's amaurosis, albinism and achromatopsia) accounted for $43 \%$ of genetic cases.

The second commonest cause of blindness in the aetiological category (Table 3) was perinatal factors, accounting for 43 children $(40 \%)$. The common causes were prematurity (14 cases), birth asphyxia (13 cases) and prematurity and birth asphyxia (five cases). This pattern of diagnosis fits that seen in other Western countries. ${ }^{16-19}$

Congenital ocular anomalies (anophthalmos and microphthalmos) accounted for $11 \%$ of children with SVI/BL. This figure is similar to findings from blind school surveys done in Japan ${ }^{20}(11.2 \%)$ and Chile ${ }^{21}$ (7\%).

ROP was responsible for visual impairment in 19 children (18\%). The number of cases of ROP has remained high at the Royal Blind School (11/99 in $1987,{ }^{4} 16 / 93$ in $1994^{5}$ ). However the youngest child seen in the current study was 9 years old, which may suggest a decreasing incidence of the disease. The introduction of cryotherapy and laser treatment for acute ROP following the CRYO ROP study ${ }^{22}$ appears to have reduced the incidence of blindness due to ROP. ${ }^{23}$ Improved monitoring of oxygen therapy in neonatal units may further reduce the incidence of ROP. ${ }^{24}$

Congenital cataract is the leading cause of surgically correctable blindness in most countries in the developing world. ${ }^{14}$ Congenital cataract was not seen as a cause of blindness in this blind school survey. Early detection and improved surgical techniques may account for this. Alternatively selection bias due to the trend towards integrated education may have produced this finding. The prevalence of visual impairment due to congenital cataract was more common in the Royal Blind School in the early 1980s $\left(12 / 99^{4}\right)$ and 1990s $\left(4 / 93^{5}\right)$. Congenital glaucoma was responsible for visual impairment in two (2\%) children. This is similar to the figures reported in other developed countries. ${ }^{18}$

The data obtained in this study indicate a changing pattern in the causes of childhood visual impairment in the Royal Blind School. There was a relative decrease in the incidence of congenital cataract leading to visual impairment. There was a decrease in the incidence of new cases of visual impairment due to retinopathy of prematurity and retinoblastoma. In each case improved detection and treatment may be responsible. Alternatively there may be selection bias due to changes in educational policy. Cerebral visual impairment, with associated neuro-developmental problems was the largest diagnostic category. Previous studies of the causes of childhood visual impairment in blind schools may have had a selection bias towards ocular disease. ${ }^{14,15}$

The WHO/PBL methodology is a useful tool for international comparisons. While this study found that the method may be used in a developed setting, further work is required. Inter-observer variation has not been measured and there is a need for validation of the forms. The method lacks sophistication in relation to cerebral visual impairment and associated handicaps. This is a deficiency when used in developed countries and in population-based studies.

Population-based studies are required in order to obtain meaningful epidemiological information. Current population-based studies in the UK (BOSU) and in Scotland (Childhood Visual Impairment in Scotland Study) which include more detailed data on cerebral visual impairment and associated impairments will address these issues.

\section{References}

1 Gilbert CE, Foster A, Thyleford B, Negrel DA. Childhood blindness: a new form of recording the causes of visual loss in children. WHO Bull 1993; 71: 485-489.

2 Rahi JS, Sripathi S, Gilbert CE, Foster A. Childhood blindness in India: causes in 1318 blind school student in nine states. Eye 1995; 9: 545-550.

3 Eckstein MB, Foster A, Gilbert CE. Causes of childhood blindness in Sri Lanka: results from children attending six schools for the blind. Br J Ophthalmol 1995; 79: 633-666.

4 Phillips CI, Levy AM, Newton M, Stokoe NL. Blindness in school children: importance of heredity, congenital cataract and prematurity. Br J Ophthalmol 1987; 71: 578584.

5 Fleck BW, Dangata Y. Causes of visual handicap in the Royal Blind School, Edinburgh, 1991-92. Br J Ophthalmol 1994; 78: 421.

6 Rosenbert T, Flage T, Hamen E et al. Incidence of registered visual impairment in the Nordic child population. Br J Ophthalmol 1996; 80: 49-53.

7 Thylefors B. A global initiative for elimination of avoidable blindness. Am J Ophthalmol 1998; 125: 90-93.

8 Foster A, Gilbert C. Epidemiology of childhood blindness. Eye 1992; 6: 173-176.

9 Gilbert CE, Canovas R, Hagan M, Rao S, Foster A. Causes of childhood blindness: results from West Africa, South India and Chile. Eye 1993; 7: 184-188.

10 Faal H, Minassian D, Sowa S, Foster A. National survey of blindness and low vision in the Gambia. $\mathrm{Br} J$ Ophthalmol 1989; 73: 82-87.

11 Foster A, Sommer A. Childhood blindness and corneal ulceration in Africa. WHO 1986; 65: 619-623.

12 Dutton GN. Cognitive visual dysfunction. Br J Ophthalmol 1994; 78: 723-726.

13 Dutton GN, Day RE, McCulloch DL. Who is a visually impaired child? A model is needed to address this 
question for children with cerebral visual impairment. Develop Med Child Neurol 1999; 41: 212-213.

14 Rogers NK, Gilbert CE, Foster A, Basit O. Childhood blindness in Uzbekistan. Eye 1995; 13: 65-70.

15 Hornby SJ, Xiao Y, Gilbert CE, Foster A, Want X, Liang X et al. Causes of childhood blindness in the People's Republic of China: results from 1131 blind school students in 18 provinces. Br J Ophthalmol 1999; 83: 929 932.

16 Fraser GR, Friedmann AJ. The causes of blindness in childhood: a study of 776 children with severe visual handicap. Johns Hopkins Press: Baltimore 1967.

17 Rosenbert T, Flage T, Hansen E, Rudenko S, Viggorson G, Rise R. Visual impairment in Nordic children. Acta Ophthalmol 1992; 70: 155-156.

18 Jay B. Causes of blindness in school children. BMJ 1987; 291: $1183-1184$
19 Goggin M, O'Keefe M. Childhood blindness in the Republic of Ireland: a national survey. $\mathrm{Br}$ J Ophthalmol 1991; 75: 425-429.

20 Fujika K, Makajima A, Yasuda N, Tanaka U, Kabasawa K. Genetic analysis of microphthalmos. Ophthalmic Paed $\mathcal{E}$ Genet 1982; 1: 139-149.

21 Gilbert C, Canova K, de Canova R, Foster A. Causes of blindness and severe visual impairment in children in Chile. Devel Child Neurol 1994; 36: 326-333.

22 CRYO-ROP group. Multicentre trial of cryotherapy for retinopathy of prematurity. Arch Ophthalmol 190; 108: 195-204

23 Fleck BW. Therapy for retinopathy of prematurity. Lancet 1999; 353: 166-167.

24 Cunnngham S, Fleck BW, Elton RA, McIntosh N. Transcutaneous oxygen levels in retinopathy of prenaturity. Lancet 1995; 346: 464-465. 